\title{
扣件支撑长度对车轮-轨道耦合振动的影响"
}

\author{
关庆华 张丹熙 王 鹏 温泽峰 金学松 \\ (西南交通大学牟引动力国家重点实验室 成都 610031)
}

\begin{abstract}
摘要: 根据轨道结构的链式特征, 提出考虑扣件支撑长度的车轮-轨道系统振动的传递矩阵分析方法。对轨道系统的垂向固有 振动特性、簧下质量作用时的轮轨垂向振动特性及扣件支撑长度的影响机理进行了分析, 结果表明, 轨道系统的第 $1 、 2$ 固 有振动模态分别为钢轨和轨枕的同相和反相共振, 其频响特征由钢轨抗弯刚度和分布质量、轨枕质量及扣件和道床的刚度和 阻尼决定, 不受扣件支撑长度的影响。扣件支撑长度对钢轨 pinned-pinned 共振和反共振影响显著, 随着扣件支撑长度的增加, 钢轨的 pinned-pinned 共振和反共振效应逐渐削弱。簧下质量作用时, 轮轨系统的振动主要以 P2 共振的形式出现, 其频率明 显低于轨道系统的第 1 固有频率, 轨道系统的振动在 pinned-pinned 共振区受扣件支撑长度的影响显著。轮轨冲击响应分析表 明, 轮轨撞击过程中体现了明显的 P2 共振特征, 在撞击初始阶段存在高频 P1 力波动, 显著的轮轨冲击会引起以 P2 共振频 率为基频的高频振动。扣件支撑长度的增加可显著降低 pinned-pinned 共振区轮轨加速度, 但对车轮和轨枕的位移影响不显著。 关键词: 轨道; 扣件支撑长度; 簧下质量；传递矩阵法； P2 共振； pinned-pinned 共振
\end{abstract} 中图分类号: U270；U211

\section{Influence of Pad Support Length on the Vibration of Wheel-track}

\author{
GUAN Qinghua ZHANG Danxi WANG Peng WEN Zefeng JIN Xuesong
}

(State Key Laboratory of Traction Power, Southwest Jiaotong University, Chengdu 610031)

\begin{abstract}
A transfer matrix method for analyzing the vibration of wheel-track system with finite support length of rail fastening system is proposed, due to the chain characteristic of track structure. The research on the natural frequencies of track system and wheel-track coupling system, as well as the influence of pad support length, indicate that the first two natural frequencies of the track system correspond to the same-phase and anti-phase vibration of rail and sleepers. The frequencies and responses of these two resonances depend on the bending stiffness and mass distribution of rail, mass of sleeper, as well as the stiffness and damping of fastening system and track bed. The support length of pad has no effect on these resonances. However, the pinned-pinned resonance and anti-resonance of rail are affected obviously by the support length of pad. With the increase of pad support length, the effects of pinned-pinned resonance and anti-resonance weaken dramatically. With the coupling of unspring mass and track system, the vibration of wheel-track system is mainly the P2 resonance, of which the frequency is much lower than the first natural frequency of track system. The response of rail near the pinned-pinned frequency is influenced by the support length of pad. The analysis of wheel rail impact response indicates that the dominated frequency of the vibration of wheel and rail is P2 resonance frequency as well as several high frequency fluctuations of P1 force during the initial stage. Severe wheel rail impact invokes high order harmonic vibrations with fundamental frequency of P2 resonance. The increase of pad support length reduces the responses of wheel and rail near the pinned-pinned frequency dramatically, nevertheless its influence on the wheel and sleeper displacement is negligible.
\end{abstract}

Key words: track; pad support length; unspring mass; transfer matrix method; P2 resonance; pinned-pinned resonance

\section{0 前言}

车辆轨道耦合系统的振动特性对动态轮轨力 有直接影响, 进而导致钢轨和车轮表面非均匀磨

* 国家自然科学基金(U1734201，51305360)、牵引动力国家重点实验室自 主研究课题(2017TPL T05)和中央高校基本科研业务费专项资金 (2682016CX126)资助项目。20180725 收到初稿, 20190326 收到修改稿
损以及疲劳伤损, 形成钢轨波磨和车轮多边形磨 损。同时，轮轨表面周期性磨损又会作为激扰反 馈作用于车辆轨道系统, 造成车辆和轨道系统的 振动噪声加剧, 影响乘客的乘坐舒适度; 剧烈的 轮轨耦合振动还是车辆和轨道部件疲劳破坏以及 道床变形的重要原因, 威胁行车安全。因此, 确 定车辆轨道系统的轮轨耦合振动特性不仅对轨道 结构的隔振设计至关重要, 对车辆部件的隔振设 
计以及车辆轨道系统的优化匹配设计都具有重要 意义。关于车辆轨道振动特性的研究, 已有相当 丰富的综述文献发表, 如文献[1-4]。翟婉明等 ${ }^{[2]}$ 指出弹性点支撑梁轨道模型较之连续弹性地基梁 模型更符合实际; NIELSEN 等 ${ }^{[3]}$ 分析了轮轨高频 相互作用及钢轨短波长波磨、车轮波磨及多边形 磨损的成因、影响和抑制措施; POPP 等 ${ }^{[4]}$ 指出 50 $500 \mathrm{~Hz}$ 中频范围内的轮轨振动对轮轨磨耗和轨道 部件伤损具有重要影响。

车辆运行过程中轨道系统的振动受到车辆耦合 的影响, 由于车辆的悬挂系统振动频率通常低于 3 $\mathrm{Hz}^{[5]}$, 高频轮轨作用力对其影响可忽略不计。因此, 轮轨系统的振动通常以簧下质量和轨道系统的耦合 振动来分析。在轮轨相互作用中, 轮轨非均匀表面 状态及线路不平顺引起的垂向冲击作用最为突出, 本文即以轮轨垂向耦合振动为研究对象, 但方法亦 可用以研究横向、纵向及扭转振动。簧下质量和轨 道耦合系统模型如图 1 所示, 其中, $k_{b}$ 和 $k_{p}$ 及 $c_{b}$ 和 $c_{p}$ 分别为道床及扣件的刚度和阻尼, $m_{s}$ 为单侧轨 道对应轨枕质量, $M_{U}$ 为簧下质量, $k_{H}$ 为轮轨间的 线性化 Hertz 接触刚度, $P_{o}$ 为作用在车轮上的垂向 力幅值, 其圆频率为 $\omega$ 。由于轮轨之间接触刚度很 大, 一般在 $1.0 \times 10^{9} \sim 1.5 \times 10^{9} \mathrm{~N} / \mathrm{m}$ 范围内, 当车 轮冲击钢轨时, 由接触刚度引起的高频力 $\mathrm{P} 1$ 的频率 可高达 400 1 $200 \mathrm{~Hz}^{[6]}$; 中频力 $\mathrm{P} 2$ 伴随于 $\mathrm{P} 1$ 力之 后, 为车轮、钢轨、轨枕及道床整体的同相振动, 其振动频率大约在 $20 \sim 100 \mathrm{~Hz}$ 。除冲击外, 轮轨表 面不平顺均能引起车辆轨道系统的 P2 振动。由于轨 枕对钢轨的离散支撑, 钢轨还存在以扣件支撑中心 为节点的 pinned-pinned 共振, 其中, 第一阶 pinned-pinned 共振模态振型如图 1 所示。

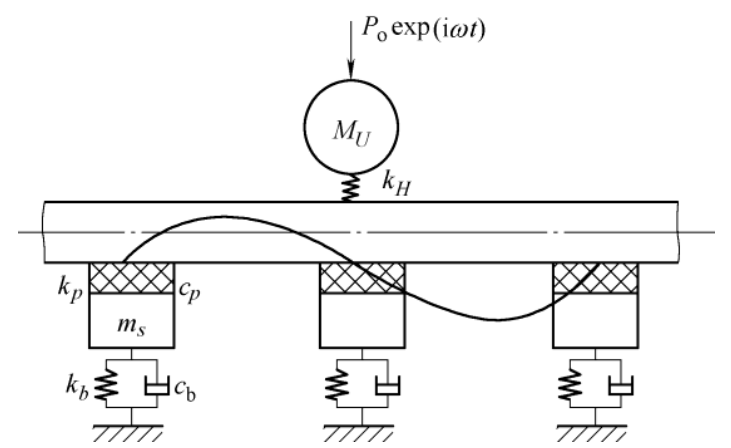

图 1 车轮-轨道耦合系统模型及 pinned-pinned 共振模态

经典的连续弹性支撑模型无法考虑离散轨枕支 撑效应, 关于轨枕的离散支撑对轨道系统振动特性 的影响, 一般通过周期结构理论 ${ }^{[7-9]}$ 、动力学 ${ }^{[5,10]}$ 及 有限元法 ${ }^{[11-12]}$ 来研究。尽管 $\mathrm{ONO}$ 等 ${ }^{[13]}$ 认为轨枕的 离散支撑引起跨中位置的钢轨 pinned-pinned 共振和
轨枕上方的反共振相互抵消, 轨道振动表现出平均 效果。对钢轨波磨的现场调查和理论研究表明, pinned-pinned 共振是导致直线及大半径曲线上短波 长钢轨波磨的主因 ${ }^{[6,14]}$, GRASSIE ${ }^{[6]}$ 称之为 “响轨” (Roaring rails)波磨。关于扣件刚度对轨道波磨的影 响机理也有不同的观点, 如 ILIAS ${ }^{[14]}$ 及 EGANA 等 ${ }^{[15]}$ 认为降低扣件刚度可以减缓钢轨波磨发展, 而谷爱 军等 ${ }^{[16]}$ 对北京地铁减振扣件轨道钢轨波磨的调查 认为, 扣件刚度和阻尼未能有效抑制特定频段的钢 轨振动是产生波磨的主因。李霞等 ${ }^{[17]}$ 认为梯形轨枕 轨道的垂横向弯曲振动与钢轨两种波长波磨的形成 有关。

目前, 我国城市轨道交通中大范围使用不同型 式的减振轨道, 出现了不同波长特征的钢轨波磨, 图 2 所示两种减振轨道出现了短波长波磨, 列车通 过时产生剧烈的振动和噪声, 并可能引起车辆和轨 道部件的疲劳断裂, 危及行车安全。因此, 有必要 深入研究轮轨耦合振动的形成机理及参数影响规 律, 而对于中高频振动, 扣件结构特征对支撑效应 的影响也不容忽视。
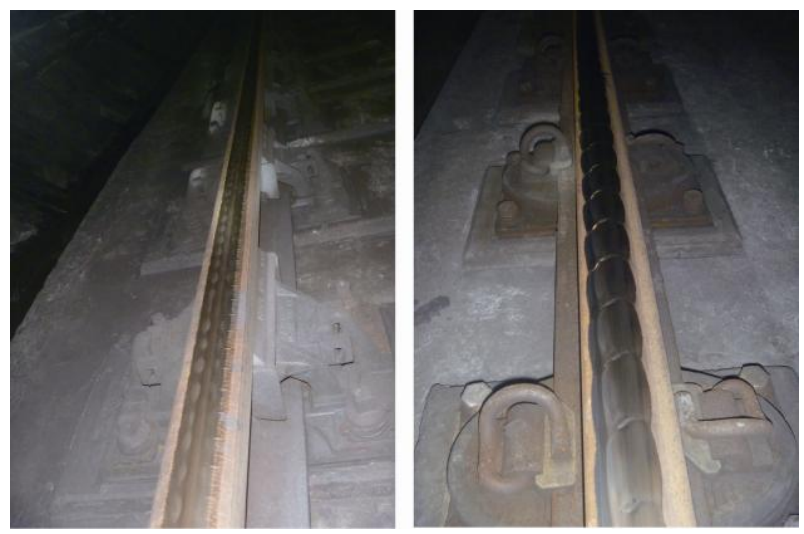

图 2 减振扣件轨道的钢轨波磨

对于扣件系统的建模, 通常以弹簧阻尼单元将 支撑中心点和钢轨连接, 王开云等 ${ }^{[18]}$ 对扣件系统进 行了细化建模, 分别考虑了扣压件及垫层的弹性特 性。而对于扣件系统沿钢轨纵向支撑长度的影响, 还少有相关研究。采用普通弹条的扣件系统, 弹性 垫板与钢轨的支撑长度接近轨枕间距的 $1 / 4$, 图 3 所示的轨道结构中扣件支撑长度接近 $200 \mathrm{~mm}$, 图 2 左侧的采用弹性支撑轨腰的先锋扣件轨道, 其扣件 有效支撑长度约为 $260 \mathrm{~mm}$, 接近于轨枕间距之半。 近年来, FERRARA 等 ${ }^{[19]}$ 和 BLANCO 等 ${ }^{[20]}$ 采用有 限元法建立的轨道系统模型考虑了扣件的支撑长 度, 研究了扣件支撑长度对轨道系统频响特性的影 响, BLANCO 等还通过时域仿真分析了不同扣件支 撑长度下的轮轨力。 


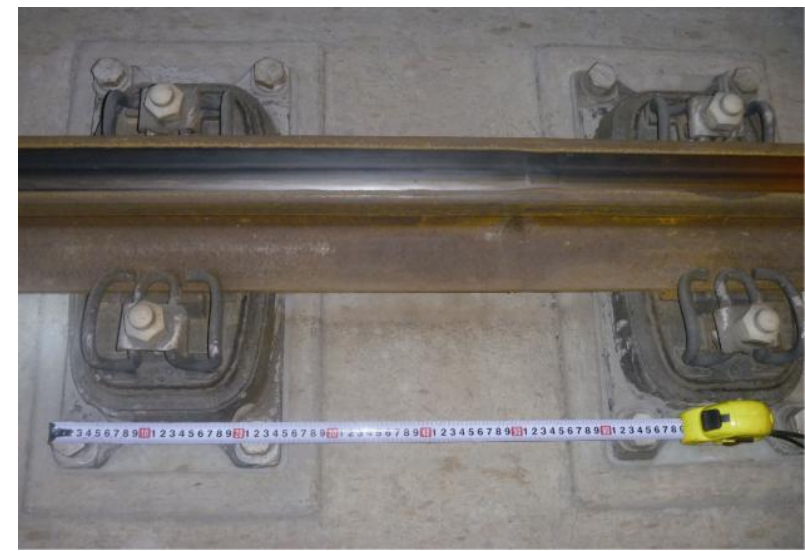

图 3 扣件及轨道结构

由于轨道系统为典型的链式结构, 扣件支撑段 钢轨可考虑为弹性基础梁, 扣件之间的钢轨可用自 由梁来模拟。本文基于传递矩阵法, 建立了簧下质 量-轨道系统振动分析模型, 可考虑不同扣件支撑长 度对轮轨振动的影响, 包括点支撑和完全连续支撑 (如嵌入式轨道), 相比于有限元法更为灵活, 计算 量小, 还可以分析簧下质量作用下轮轨系统耦合振 动特性。应用本文模型对轮轨系统的垂向振动机理 进行分析, 明确扣件支撑长度对轨道系统固有振动 特性及轮轨耦合振动特性的影响规律。基于扣件面 支撑的 Timoshenko 梁轨道模型, 对滚动车轮和轨道 的冲击响应进行了时频域分析，对比了不同扣件支 撑长度时轮轨振动和轮轨力特征。

\section{1 扣件面支撑的轨道振动分析模型}

\section{1 振动微分方程}

图 4 为考虑扣件纵向支撑长度的面支撑轨道模 型， $y 、 \theta 、 M 、 F$ 分别为钢轨截面处的垂向位移、 转角、弯矩和剪力, $l_{s 1}$ 和 $l_{s 2}$ 分别为两轨枕间钢轨悬 空的长度和扣件支撑长度, 轨枕间距 $l=l_{s 1}+l_{s 2}$ 。

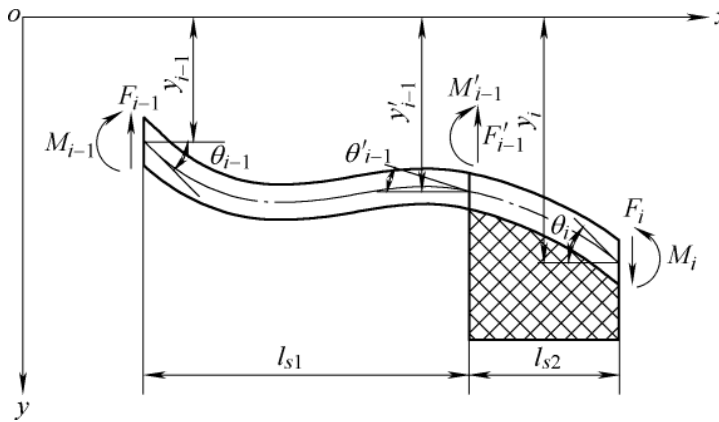

图 4 扣件面支撑钢轨的力学模型

如视一个轨跨的钢轨和轨枕组成轨道模型的一 个单元, 则整个轨道系统为一系列相同轨道单元组 成的链式结构。其中, 悬空段钢轨的垂向弯曲自由
振动以 Euler 梁运动方程表示为

$$
E I \frac{\partial^{4} y}{\partial x^{4}}+m_{r} \frac{\partial^{2} y}{\partial t^{2}}=0
$$

式中, $E I$ 为钢轨抗弯刚度, $m_{r}$ 为单位长度的钢轨质 量, 采用分离变量法, 该段钢轨振动位移可表示为

$$
\begin{gathered}
y(x, t)=\left[C_{1} S(\lambda x)+C_{2} T(\lambda x)+\right. \\
\left.C_{3} U(\lambda x)+C_{4} V(\lambda x)\right] \sin \omega t
\end{gathered}
$$

式中, $\lambda=\sqrt[4]{m_{r} \omega^{2} / E I}, \omega$ 为振动圆频率, 系数 $C_{1}$, $C_{2}, C_{3}$ 和 $C_{4}$ 由梁的边界条件确定。 $S 、 T 、 U 、 V$ 分 别表示为

$$
\left\{\begin{array}{l}
S(\lambda x)=\frac{1}{2}(\cosh \lambda x+\cos \lambda x) \\
T(\lambda x)=\frac{1}{2}(\sinh \lambda x+\sin \lambda x) \\
U(\lambda x)=\frac{1}{2}(\cosh \lambda x-\cos \lambda x) \\
V(\lambda x)=\frac{1}{2}(\sinh \lambda x-\sin \lambda x)
\end{array}\right.
$$

根据梁的转角、弯矩和剪力与弯曲变形关系

$$
\theta=\frac{\partial y}{\partial x} \quad M=-E I \frac{\partial^{2} y}{\partial x^{2}} \quad F=-E I \frac{\partial^{3} y}{\partial x^{3}}
$$

可以 $x_{i-1}$ 处梁的状态列阵表示 $x_{i-1}^{\prime}$ 处梁的状态列阵 为

$\left(\begin{array}{c}-y \\ -\theta \\ M \\ F\end{array}\right)_{i-1}^{\prime}=\left(\begin{array}{cccc}S & \frac{T}{\lambda} & \frac{U}{E I \lambda^{2}} & \frac{V}{E I \lambda^{3}} \\ \lambda V & S & \frac{T}{E I \lambda} & \frac{U}{E I \lambda^{2}} \\ E I \lambda^{2} U & E I \lambda V & S & \frac{T}{\lambda} \\ E I \lambda^{3} T & E I \lambda^{2} U & \lambda V & S\end{array}\right)\left(\begin{array}{c}-y \\ -\theta \\ M \\ F\end{array}\right)_{i-1}$

式中, $S=S\left(\lambda l_{s 1}\right), T=T\left(\lambda l_{s 1}\right), U=U\left(\lambda l_{s 1}\right), V=V\left(\lambda l_{s 1}\right)$ 。 式(5)可简写为

$$
\boldsymbol{v}_{i-1}^{\prime}=\boldsymbol{D}\left(l_{s 1}\right) \boldsymbol{v}_{i-1}
$$

扣件支撑段钢轨的垂向弯曲自由振动以 Winkler 弹性地基上的 Euler 梁运动方程表示为

$$
E I \frac{\partial^{4} y}{\partial x^{4}}+m_{r} \frac{\partial^{2} y}{\partial t^{2}}+k y=0
$$

式中, $k$ 为钢轨基础弹性系数, 其由扣件及道床的 刚度和阻尼，以及对应轨枕质量决定，以动刚度形 式表示为

$$
k=\frac{1}{l_{s 2}} \frac{\left(-m_{s} \omega^{2}+\mathrm{i} \omega c_{b}+k_{b}\right)\left(k_{p}+\mathrm{i} \omega c_{p}\right)}{-m_{s} \omega^{2}+\mathrm{i} \omega\left(c_{b}+c_{p}\right)+\left(k_{b}+k_{p}\right)}
$$

式中, $i$ 为单位虚数。 
同理，扣件支撑段钢轨振动位移可表示为

$$
\begin{gathered}
y(x, t)=\left[C_{1}^{*} S\left(\lambda^{*} x\right)+C_{2}^{*} T\left(\lambda^{*} x\right)+\right. \\
\left.C_{3}^{*} U\left(\lambda^{*} x\right)+C_{4}^{*} V\left(\lambda^{*} x\right)\right] \sin \omega t
\end{gathered}
$$

式中, $\lambda^{*}=\sqrt[4]{\left(m_{r} \omega^{2}-k\right) / E I}$, 系数 $C_{1}^{*} 、 C_{2}^{*} 、 C_{3}^{*}$ 和 $C_{4}^{*}$ 由梁的边界条件确定。

类似地，扣件支撑段钢轨状态列阵传递关系为

$$
\boldsymbol{v}_{i}=\boldsymbol{E}\left(l_{s 2}\right) \boldsymbol{v}_{i-1}^{\prime}
$$

式中, 传递矩阵 $\boldsymbol{E}$ 和 $\boldsymbol{D}$ 具有相同的形式, 不同的是 矩阵 $\boldsymbol{E}$ 中以 $\lambda^{*}$ 代替 $\lambda, l_{s 2}$ 代替 $l_{s 1}$ 。

由式(5)、(9)得到一个轨道单元前后的状态列阵 传递关系为

$$
\boldsymbol{v}_{i}=\boldsymbol{E}\left(l_{s 2}\right) \boldsymbol{D}\left(l_{s 1}\right) \boldsymbol{v}_{i-1}=\boldsymbol{T} \boldsymbol{v}_{i-1}
$$

式中, $\boldsymbol{T}$ 即为轨道单元钢轨状态列阵的传递矩阵。

\section{2 传递矩阵特性}

若以某悬空段钢轨左侧截面状态列阵为初始量 $\boldsymbol{v}_{0}$, 则传递至第 $n$ 个扣件右侧钢轨截面处的状态列 阵可表示为

$$
\boldsymbol{v}_{n}=\boldsymbol{T}^{n} \boldsymbol{v}_{0}
$$

传递矩阵 $\boldsymbol{T}$ 的特征值和特征矢量可通过如下特 征方程来求解

$$
(\boldsymbol{T}-u \boldsymbol{I}) \boldsymbol{\varphi}=0
$$

整理得到特征值的四次方程

$$
u^{4}+I_{1} u^{3}+I_{2} u^{2}+I_{1} u+1=0
$$

式中, $I_{1}=-\operatorname{tr}(\boldsymbol{T}), I_{2}=\sum_{i+1 \leqslant j=2}^{4}\left(t_{i i} t_{j j}-t_{i j} t_{j i}\right), t_{i j}=\boldsymbol{T}(i, j)$ 。

根据周期结构传递矩阵特征值特性 ${ }^{[21]}$, 由式(13) 可求得两对互为倒数的复特征值 $u_{j}$ 和复特征矢量 $\boldsymbol{\varphi}_{j}, j=1 \sim 4$, 若取 $\left|u_{1}\right|$ 和 $\left|u_{2}\right|$ 小于 1 , 则 $u_{3}=1 / u_{2}, u_{4}=1 / u_{1}$ 。 式(11)可表示为

$$
\boldsymbol{v}_{n}=\sum_{j=1}^{4} A_{j} u_{j}^{n} \boldsymbol{\varphi}_{j}
$$

式中, $A_{j}$ 为待定系数。

钢轨受初始激励引起的振动波为衰减波, 经过 足够长距离衰减后, 钢轨状态列阵元素均应趋近于 零, 式(14)中系数 $A_{3}=A_{4}=0$, 整理可得

$$
\left(\begin{array}{l}
M \\
F
\end{array}\right)_{n}=\boldsymbol{H}\left(\begin{array}{c}
-y \\
-\theta
\end{array}\right)_{n}
$$

式中, $\boldsymbol{H}=\left(\begin{array}{ll}\varphi_{31} & \varphi_{32} \\ \varphi_{41} & \varphi_{42}\end{array}\right)\left(\begin{array}{ll}\varphi_{11} & \varphi_{12} \\ \varphi_{21} & \varphi_{22}\end{array}\right)^{-1}, \varphi_{i j}=\boldsymbol{\varphi}_{j}(i)$ 。

\section{3 轨道振动导纳}

如图 5 所示，钢轨某点承受单位简谐激励时， 其激励点处钢轨状态列阵 $\boldsymbol{v}_{0}^{R}$ 与激励点右侧第 1 个 扣件支撑端右端钢轨截面的状态列阵 $\boldsymbol{v}_{1}^{R}$ 的传递关 系可表示为

$$
\boldsymbol{v}_{0}^{R}=\boldsymbol{R} \boldsymbol{v}_{1}^{R}
$$

式中, $\boldsymbol{R}=\left\{\begin{array}{ll}\boldsymbol{D}^{-1}\left(l_{s 1}-x_{a}\right) \boldsymbol{E}^{-1}\left(l_{s 2}\right) & 0 \leqslant x_{a} \leqslant l_{s 1} \\ \boldsymbol{E}^{-1}\left(l_{s 1}+l_{s 2}-x_{a}\right) & l_{s 1}<x_{a} \leqslant l_{s 1}+l_{s 2}\end{array}\right.$, 矩阵 $\boldsymbol{R}$ 可表示为 4 个 $2 \times 2$ 的分块矩阵 $\boldsymbol{R}=\left(\begin{array}{ll}\boldsymbol{R}_{11} & \boldsymbol{R}_{12} \\ \boldsymbol{R}_{21} & \boldsymbol{R}_{22}\end{array}\right)$ 。

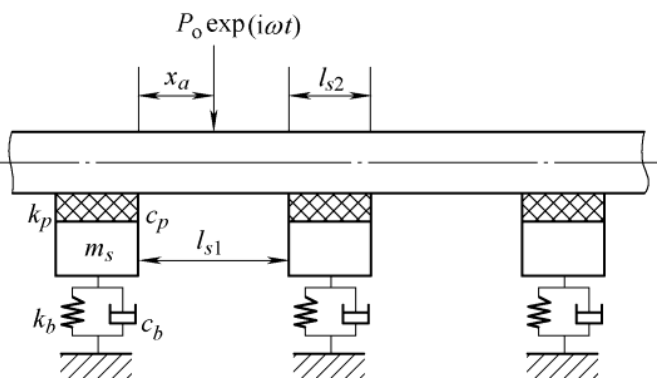

图 5 轨道系统模型及激励载荷示意图

激励点右侧最近的扣件支撑段右端钢轨状态列 阵满足式(15), 根据式(16)可得到激励点处钢轨右侧 截面弯矩、剪力与弯曲位移和转角间关系为

$$
\left(\begin{array}{l}
M \\
F
\end{array}\right)_{0}^{R}=\left(\boldsymbol{R}_{21}+\boldsymbol{R}_{22} \boldsymbol{H}_{R}\right)\left(\boldsymbol{R}_{11}+\boldsymbol{R}_{12} \boldsymbol{H}_{R}\right)^{-1}\left(\begin{array}{r}
-y \\
-\theta
\end{array}\right)_{0}^{R}
$$

式中, $\boldsymbol{H}_{R}$ 为右向刚度矩阵, 由式(15)确定。

同理, 轨道激励点处左侧钢轨状态列阵 $v_{0}^{L}$ 与激 励点左侧第 1 个扣件支撑段左端钢轨截面的状态列 阵 $\boldsymbol{v}_{-1}^{L}$ 传递关系可表示为

$$
\boldsymbol{v}_{0}^{L}=\boldsymbol{L} \boldsymbol{v}_{-1}^{L}
$$

式中, $\boldsymbol{L}=\left\{\begin{array}{ll}\boldsymbol{D}\left(x_{a}\right) \boldsymbol{E}\left(l_{s 2}\right) & 0 \leqslant x_{a} \leqslant l_{s 1} \\ \boldsymbol{E}\left(x_{a}-l_{s 1}\right) \boldsymbol{D}\left(l_{s 1}\right) \boldsymbol{E}\left(l_{s 2}\right) & l_{s 1}<x_{a} \leqslant l_{s 1}+l_{s 2}\end{array}\right.$ 。

根据式(15)、(18), 可得激励点钢轨左截面状态 元素关系为

$$
\left(\begin{array}{l}
M \\
F
\end{array}\right)_{0}^{L}=\left(\boldsymbol{L}_{21}+\boldsymbol{L}_{22} \boldsymbol{H}_{L}\right)\left(\boldsymbol{L}_{11}+\boldsymbol{L}_{12} \boldsymbol{H}_{L}\right)^{-1}\left(\begin{array}{c}
-y \\
-\theta
\end{array}\right)_{0}^{L}
$$

式中, 左向刚度矩阵 $\boldsymbol{H}_{L}$ 由式(15)类似方法确定，同 式(16), $\boldsymbol{L}_{11} 、 \boldsymbol{L}_{12} 、 \boldsymbol{L}_{21}$ 和 $\boldsymbol{L}_{22}$ 为 $\boldsymbol{L}$ 的 4 个分块矩阵。 根据激励点处左右截面状态平衡条件：弯曲位 移、转角和弯矩相等, 剪力满足 $F_{0}^{L}-P=F_{0}^{R}$, 得

$$
\left(\begin{array}{l}
M \\
F
\end{array}\right)_{0}^{L}-\left(\begin{array}{l}
M \\
F
\end{array}\right)_{0}^{R}=\left(\begin{array}{l}
0 \\
P
\end{array}\right)
$$


将式(17)、(19)代入式(20), 可得到不同激励频 率下激励点处钢轨弯曲位移和转角随激振载荷 $P$ 的 原点导纳。

\section{2 扣件支撑长度对轨道自振的影响}

根据上述理论对不同扣件支撑长度的轨道系统 固有振动特性进行对比分析, 明确轨道系统的各振 动主频的产生机理及扣件支撑长度的影响。

\section{1 扣件支撑长度对轨道第 $1 、 2$ 共振频率的影响}

轨道系统参数如表 1 所示, 其中钢轨质量和抗 弯刚度取 CN60 钢轨参数, 扣件及道床的刚度和阻 尼及轨枕质量参数同文献 $[10,20]$, 扣件支撑长度选 取 $0 \mathrm{~m}, 0.16 \mathrm{~m}, 0.27 \mathrm{~m}$ 及 $0.6 \mathrm{~m}$, 分别代表点支撑、 有限长度支撑和完全连续支撑。

\section{表 1 轨道系统计算参数}

\begin{tabular}{lc||lc}
\hline \multicolumn{1}{c|}{ 参数 } & 数值 & \multicolumn{1}{c}{ 参数 } & 数值 \\
\hline$m_{r} /(\mathrm{kg} / \mathrm{m})$ & 60.6 & $E I /\left(\mathrm{N} \cdot \mathrm{m}^{2}\right)$ & $6.63 \times 10^{6}$ \\
$l / \mathrm{m}$ & 0.6 & $l_{s 2} / \mathrm{m}$ & $0 / 0.16 / 0.27 / 0.6$ \\
$k_{p} /(\mathrm{N} / \mathrm{m})$ & $3.5 \times 10^{8}$ & $c_{p} /(\mathrm{N} \cdot \mathrm{s} / \mathrm{m})$ & $4.8 \times 10^{4}$ \\
$k_{b} /(\mathrm{N} / \mathrm{m})$ & $0.7 \times 10^{8}$ & $c_{p} /(\mathrm{N} \cdot \mathrm{s} / \mathrm{m})$ & $4.7 \times 10^{4}$ \\
$m_{s} / \mathrm{kg}$ & 160 & $k_{H} /(\mathrm{N} / \mathrm{m})$ & $1.028 \times 10^{9}$ \\
\hline
\end{tabular}

图 6 为不同扣件支撑长度下的轨道系统受跨中 及轨枕上方激励时的钢轨位移导纳曲线。由图可知, 不同位置激励下的轨道系统在 $1000 \mathrm{~Hz}$ 内存在 $90 \mathrm{~Hz}$ 和 $529 \mathrm{~Hz} 2$ 个显著的共振峰, 分别对应于钢轨和轨 枕的同相共振和反相共振, 跨中和轨枕上方激励的 反共振频率分别为 $252 \mathrm{~Hz}$ 和 $270 \mathrm{~Hz}$; 高于 $1000 \mathrm{~Hz}$ 时轨道系统存在 pinned-pinned 共振及反共振区, 其 中, 跨中激励为共振区, 轨枕上方激励为反共振区, 反共振频率高于共振频率。显然, 扣件支撑长度对 pinned-pinned 共振和反共振影响显著, 而对前 2 个 共振峰的频率及响应幅值均无明显影响。

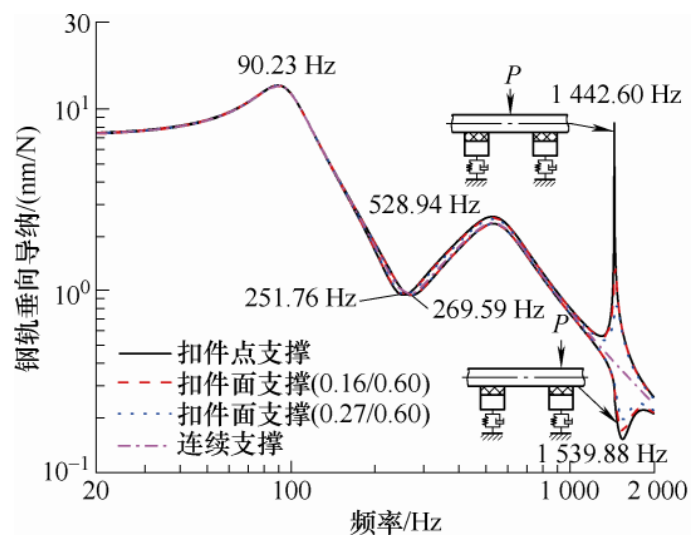

图 6 不同扣件支撑类型下钢轨垂向导纳

文献[10]按 UIC60 钢轨参数计算的轨道系统第
1、2 主频分别为 $95 \mathrm{~Hz}$ 和 $550 \mathrm{~Hz}$, 反共振频率为 $260 \mathrm{~Hz}$, 与本文根据 CN60 钢轨参数的计算结果基 本一致，表明了本文模型和计算结果的合理性。

为明确轨道系统第 $1 、 2$ 共振区的形成机理, 暂不考虑钢轨的抗弯刚度, 可将轨道系统简化为 图 7 所示的 2 自由度振系, 图中 $m_{r s}$ 为扣件支撑单 元的钢轨分布质量。根据振动理论, 该振系具有 2 阶固有振动频率，忽略扣件和道床阻尼后可表示 为 ${ }^{[22]}$

$$
\begin{aligned}
& \omega_{1,2}^{2}=\frac{1}{2}\left[\omega_{s}^{2}+\omega_{r s}^{2}\left(1+\frac{m_{r s}}{m_{s}}\right)\right] \pm \\
& \frac{1}{2} \sqrt{\left[\omega_{s}^{2}+\omega_{r s}^{2}\left(1+\frac{m_{r s}}{m_{s}}\right)\right]^{2}-4 \omega_{s}^{2} \omega_{r s}^{2}}
\end{aligned}
$$

式中, $\omega_{s}^{2}=k_{b} / m_{s}, \omega_{r s}^{2}=k_{p} / m_{r s}$, 频率较低的 $\omega_{1}$ 为 轨道系统的自振基频, 频率较高的 $\omega_{2}$ 为轨道系统的 高阶振动圆频率。

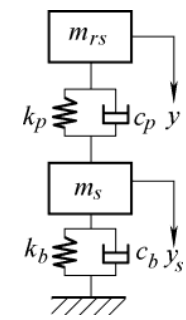

图 7 轨道系统简化模型(不考虑钢轨抗弯刚度)

根据 2 自由度振系特性, 不考虑阻尼的轨道系 统在 $\omega_{1}$ 和 $\omega_{2}$ 间存在一个反共振频率

$$
\tilde{\omega}_{1}^{2}=\left(k_{b}+k_{p}\right) / m_{s}
$$

在该频率处, 钢轨静止不动, 轨枕以 $\tilde{\omega}_{1}$ 角频率振动。

以表 1 中轨道参数为例, 轨枕在道床刚度上的 自振频率为 $105.27 \mathrm{~Hz}$, 钢轨分布质量在扣件刚度上 的自振频率为 $493.79 \mathrm{~Hz}$ 。根据式(21)、(22)计算得 到, 轨道系统的低阶和高阶振动频率分别为 $f_{1}=94.69$ $\mathrm{Hz}, f_{2}=548.96 \mathrm{~Hz}$, 反共振频率为 $257.86 \mathrm{~Hz}$ 。因此, 不考虑钢轨抗弯刚度时的轨道系统第 1 、2 共振频率 略高于图 4 中考虑钢轨抗弯刚度时的第 1 、2 共振频 率 $(90.26 \mathrm{~Hz}$ 和 $528.94 \mathrm{~Hz})$ 。

不考虑阻尼的轨道系统中钢轨和轨枕振幅比为

$$
r_{i}=\frac{Y^{(i)}}{Y_{s}^{(i)}}=\frac{\tilde{\omega}_{1}^{2}-\omega_{i}^{2}}{k_{p} / m_{s}} \quad i=1,2
$$

由式(23)可知, 低阶振动中 $(i=1)$, 钢轨和轨 枕振幅比为正; 高阶振动中 $(i=2)$, 钢轨和轨枕振 幅比为负, 钢轨和轨枕作反相振动, 在钢轨和轨 枕间存在一个振动节点, 任何振动瞬时该节点保 持静止。由于高阶振动中节点的存在限制了钢轨 
和轨枕振幅增大, 轨道系统的振动能量主要集中 于低阶振动。

考虑扣件阻尼和道床阻尼后, 2 自由度轨道模 型受钢轨激扰力作用时, 其钢轨和轨枕导纳曲线如 图 8 所示, 标注 $E I=0$ 的为不考虑钢轨抗弯刚度的 2 自由度振系导纳曲线, 未标注的为通过传递矩阵 法得到的钢轨和轨枕导纳曲线。由图可知, 考虑扣 件和道床阻尼后, 不考虑钢轨抗弯刚度的 2 自由度 模型得到的轨道系统振动主频和通过传递矩阵法得 到的轨道系统主频一致, 均略低于不考虑阻尼时的 振动主频, 不同的是 2 自由度模型由于未考虑钢轨 抗弯刚度对钢轨弯曲的限制, 其得到的响应幅值高 于考虑了钢轨抗弯刚度的实际轨道系统。

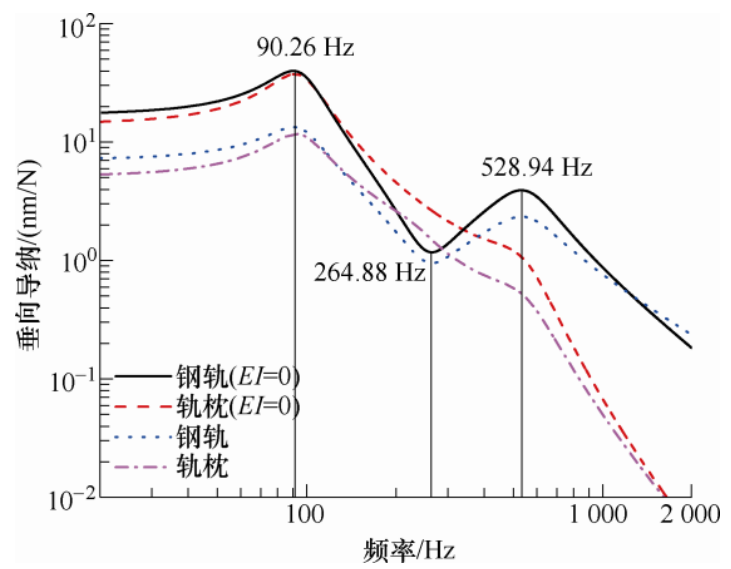

图 8 简化轨道模型和完整轨道模型的钢轨和轨枕垂向导纳

以上分析表明, 轨道系统第 $1 、 2$ 共振区与钢轨 抗弯刚度和质量、扣件刚度和阻尼、轨枕质量及道 床刚度和阻尼有关，在同样的扣件总刚度条件下， 与扣件支撑长度无关。

\section{2 扣件支撑长度对轨道 pinned-pinned 共振响应 的影响}

图 9 为不同扣件支撑长度下的轨道系统受跨中 及轨枕上方激励时钢轨的 pinned-pinned 振动特性。 由图可知, 将扣件支撑考虑为点支撑时, 跨中激励

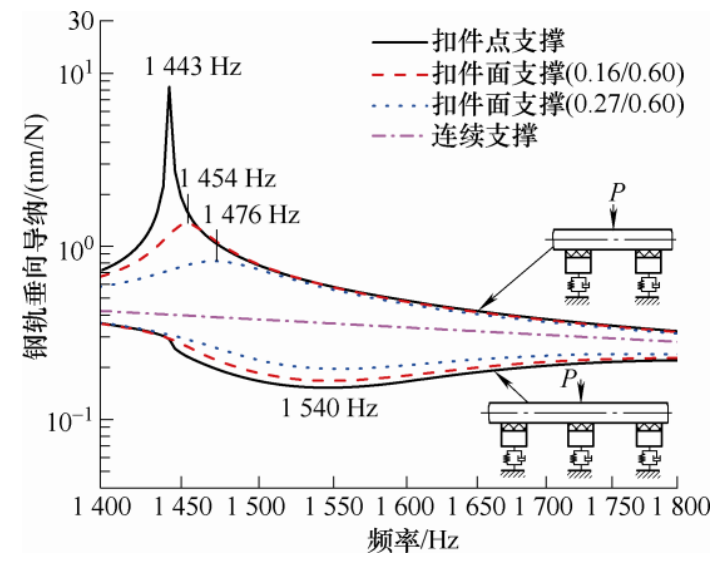

图 9 不同扣件支撑长度下钢轨 pinned-pinned 共振
时钢轨上存在显著的共振峰值, 图 9 中所示单位载 荷激励下，点支撑时钢轨位移响应幅值为 $8.35 \mathrm{~nm}$, 而当扣件支撑长度增加时, pinned-pinned 共振峰显 著下降, 当扣件长度为 $0.16 \mathrm{~m}$ 和 $0.27 \mathrm{~m}$ 时, 钢轨位 移响应幅值分别为 $1.17 \mathrm{~nm}$ 和 $0.73 \mathrm{~nm}$, 若轨道完全 连续支撑， pinned-pinned 共振消失，钢轨响应幅值 为 $0.40 \mathrm{~nm}$ 。此外, 随着扣件支撑长度的增加, pinned-pinned 共振频率略有提高。

由于轨道系统在扣件约束下的 pinned-pinned 共 振波长为两个轨枕枕距的长度, 扣件支撑位置为其 振动节点，当激励位于轨枕上方时，将破坏其节点 特征而出现反共振。如图 9 所示, 当考虑扣件点支 撑时, 反共振峰处钢轨位移响应幅值为 $0.15 \mathrm{~nm}$, 扣 件支撑长度为 $0.16 \mathrm{~m}$ 和 $0.27 \mathrm{~m}$ 时, 反共振响应幅值 为 $0.17 \mathrm{~nm}$ 和 $0.19 \mathrm{~nm}$, 在轨道完全连续支撑时, 反 共振峰消失。

\section{3 扣件支撑长度对轨枕响应的影响}

图 10 为不同扣件支撑长度下的轨枕响应曲线。 图中可知，尽管不同扣件支撑长度对轨道 pinnedpinned 共振响应显著, 但在 $2000 \mathrm{~Hz}$ 以内频率范围 内轨枕的响应几乎不受扣件支撑长度的影响，显著 共振峰出现在轨道系统的第 1 共振区。

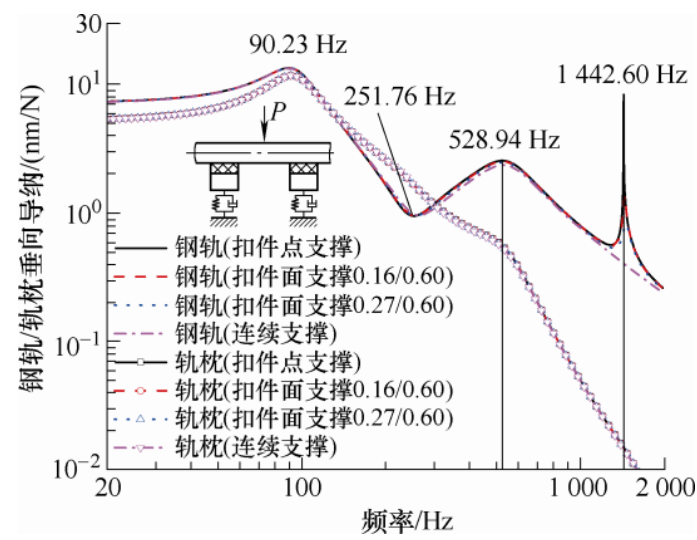

图 10 扣件支撑长度对轨枕垂向导纳的影响

图中还表明，在激振频率高于第 1 共振频率低 于第 2 共振频率范围内存在轨枕响应高于钢轨响应 的轨枕活跃区, 按表 1 参数计算的频率范围为 120 $300 \mathrm{~Hz}$ 。

\section{3 扣件支撑长度对轮轨耦合共振的影响}

\section{1 簧下质量-轨道系统的耦合振动特性}

图 11 为簧下质量-轨道系统耦合振动特性, 扣 件和钢轨间视为点支撑, 轨道参数如表 1 所示, $M_{U}=$ $550 \mathrm{~kg}$, 图中以车轮代称。由图 11a 位移响应曲线 可知，与簧下质量相互作用时，轨道系统的第 1 共 
振峰的响应要显著突出于第 2 共振峰和 pinnedpinned 共振峰。轨道系统的第 1 共振频率由 $90.23 \mathrm{~Hz}$ 降为 $62.65 \mathrm{~Hz}$, 为簧下质量和轨道系统的 P2 共振频 率; 第 2 共振频率降为 $235.71 \mathrm{~Hz}$, 位于轨枕振动显 著区; pinned-pinned 共振频率略有提高。从图 $11 \mathrm{~b}$ 的加速度响应曲线来看, 簧下质量-轨道系统振动峰 值仍体现在 P2 共振、第 2 共振峰及 pinned-pinned 共振峰, 峰值频率略高于位移响应的峰值频率, 但 高于 P2 共振频率时加速度响应幅值变化不大, 这是 由于在高频范围内振动响应由惯性主导, 加速度响 应突出而位移几乎不变。从图中还可知, pinnedpinned 共振频率处簧下质量的位移导纳和加速度导 纳均未出现明显的峰值或者谷值。因此, pinnedpinned 共振对钢轨振动影响最为显著, 轨枕次之, 对车轮振动的影响很小。

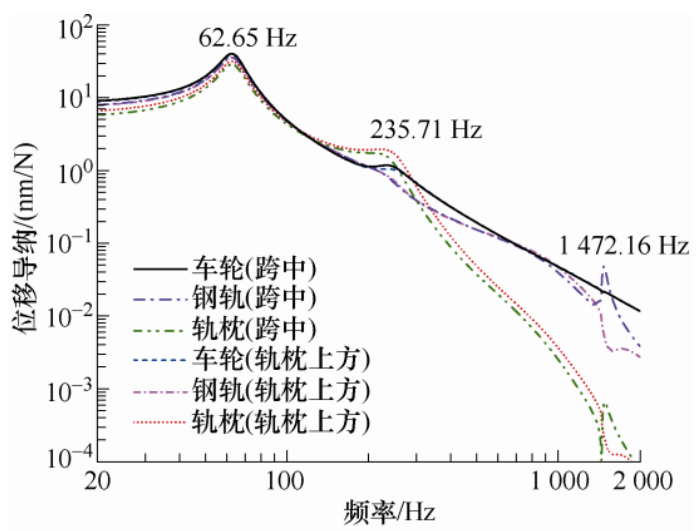

(a) 车轮、钢轨和轨枕的位移导纳

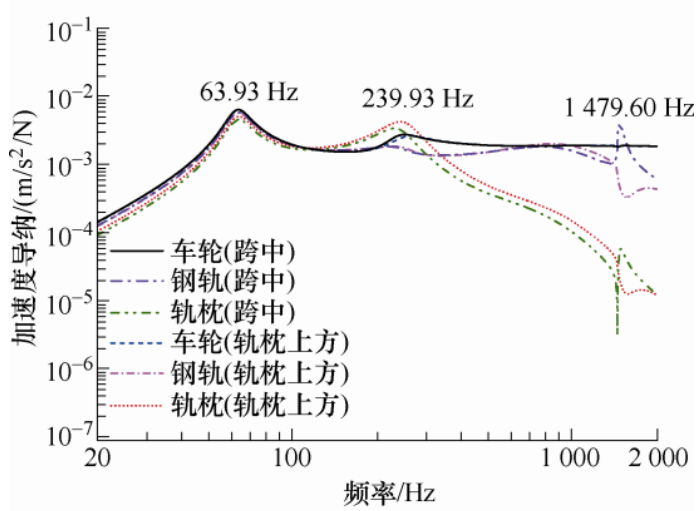

(b) 车轮、钢轨和轨枕的加速度导纳

图 11 扣件点支撑时簧下质量-轨道系统振动特性

\section{2 扣件支撑长度对簧下质量-轨道系统振动的影响}

图 12 为不同扣件支撑长度时簧下质量-轨道系 统耦合振动特性。由图可知, 不同扣件支撑长度对 $1000 \mathrm{~Hz}$ 以内的轨道振动位移响应无影响, 对 pinned-pinned 共振区及反共振区有显著影响, 随扣 件支撑长度的增加, pinned-pinned 共振和反共振影 响逐渐减弱。与图 11 中类似, 扣件支撑长度对簧下 质量的振动响应影响很小。

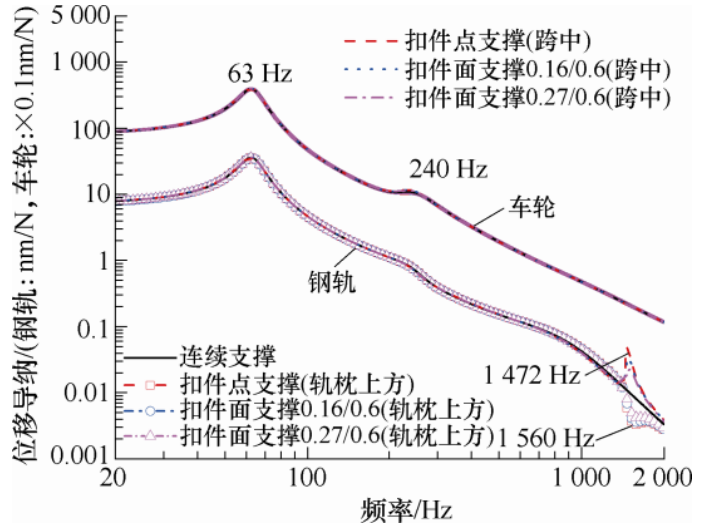

图 12 不同扣件支撑长度时簧下质量-轨道系统振动响应

\section{4 轮轨冲击响应分析}

为进一步分析不同扣件支撑长度下轮轨系统的 动力学特征, 通过模态叠加法对有限长轨道受车轮 冲击时的位移和加速度及轮轨力特征进行了分析。 轨道长度为 $24 \mathrm{~m}$, 车辆行驶速度为 $60 \mathrm{~km} / \mathrm{h}$, 其他 参数如表 1 所示。轮轨高频冲击时, 由于 Euler 梁 模型未考虑剪切变形和转动惯量的影响会造成较大 的误差, 为更真实地体现轮轨高频冲击行为, 轨道 模型采用了 Timoshenko 梁模型。需要指出的是, 此 处的完全连续支撑实际为扣件支撑长度等于轨枕间 距，轨枕仍以等间距离散分布。

\section{1 扣件支撑长度对轮轨动力响应的影响}

图 13 为车轮以 $0.2 \mathrm{~m} / \mathrm{s}$ 速度冲击钢轨时的车轮 和钢轨垂向位移, 其中, 线条为车轮位移, 带符号 线为钢轨位移，下文类似。由图可知，不同扣件支 撑长度对首次冲击过程中的车轮位移峰值无明显影 响, 但钢轨完全连续支撑时响应较小。平衡状态时, 随扣件支撑长度的增加, 轮轨垂向下沉位移略有减 小，最为显著的为完全连续支撑钢轨。

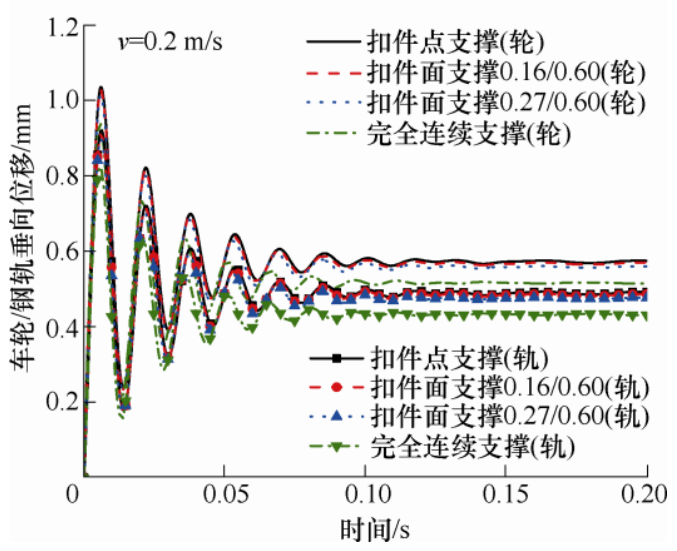

图 13 不同扣件支撑长度时簧下质量-轨道系统冲击响应

图 14 为车轮以 $1 \mathrm{~m} / \mathrm{s}$ 速度冲击钢轨时的车轮和 
钢轨接触力曲线。由图可知, 不同扣件支撑长度对 轮轨冲击力无明显影响。在轮轨碰撞的初始阶段, 由于车轮相对钢轨的速度较高, 存在高频的轮轨冲 击力 (P1 力), 经历几次高频波动后, 轮轨力随同轮 轨位移以较低频率波动( $\mathrm{P} 2$ 力)。

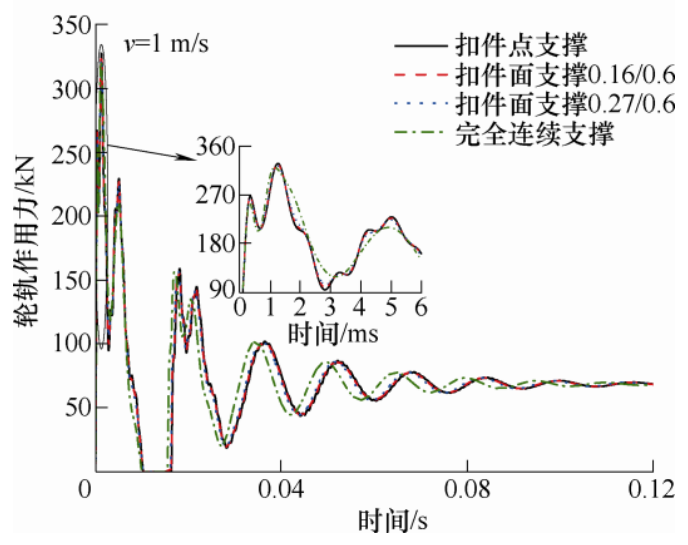

图 14 不同扣件支撑长度时的轮轨作用力

\section{2 扣件支撑长度对轮轨动力频响的影响}

图 15 为车轮以两种速度冲击钢轨时的轮轨力 功率谱密度曲线。由图可知, 当轮轨冲击速度较低 时, 轮轨力的第 1 主频为 $63 \mathrm{~Hz}$, 为 $\mathrm{P} 2$ 共振频率, 同时在 $260 \mathrm{~Hz}$ 附近及 pinned-pinned 共振区也出现 了轮轨力峰值。当轮轨碰撞速度较高时, 轮轨力仍

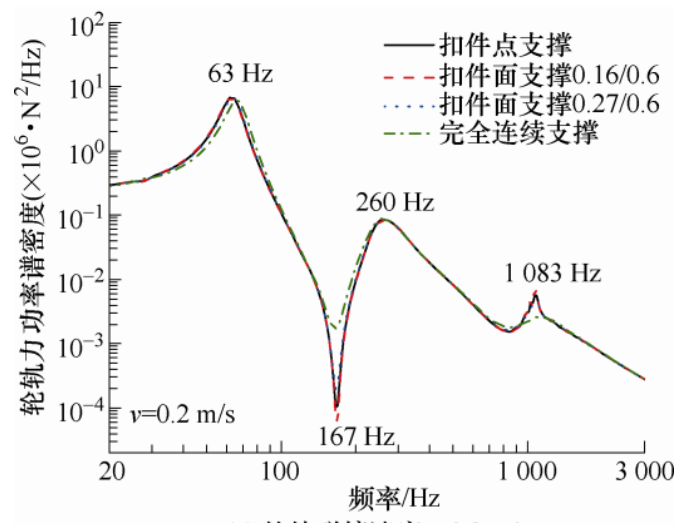

(a) 轮轨碰撞速度 $v=0.2 \mathrm{~m} / \mathrm{s}$

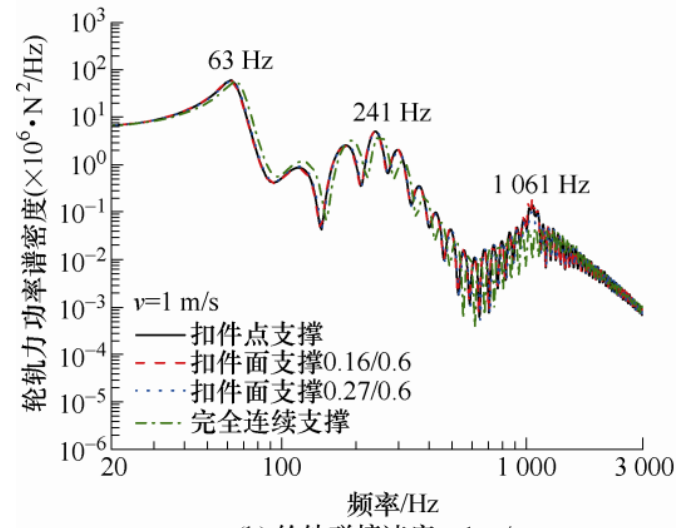

(b) 轮轨碰撞速度 $v=1 \mathrm{~m} / \mathrm{s}$

图 15 不同扣件支撑长度时轮轨力功率谱密度曲线
分布在上述 3 个主频区，不同的是，整个频率范围 内存在以 $\mathrm{P} 2$ 共振频率为基频的高次谐波振动。图中 结果显示, 增加扣件支撑长度可降低 pinned-pinned 共振时轮轨接触力, 与前文不同的是, 钢轨完全连 续支撑实际为扣件完全连续支撑，轨枕仍以等间距 分布, 所以在 pinned-pinned 共振频率处存在一定的 轮轨力峰值。

对比图 6 和图 15, 图中 pinned-pinned 共振频率 有较大差异, 这是由于前文传递矩阵分析中采用了 Euler 梁模型, 而此处轮轨冲击分析中采用了 Timoshenko 梁模型, 两种梁模型的 pinned-pinned 共振频率为

$$
\left\{\begin{array}{l}
f_{p p}^{E-B}=\frac{\pi}{2 l^{2}} \sqrt{\frac{E I}{m_{r}}} \\
f_{p p}^{T-B}=\frac{1}{2 \pi} \sqrt{\frac{b-\sqrt{b^{2}-4 a c}}{2 a}}
\end{array}\right.
$$

式中, $f_{p p}^{E-B}$ 和 $f_{p p}^{T-B}$ 分别为 Euler 梁和 Timoshenko 梁钢轨的 pinned-pinned 共振频率, $a=m_{r} J_{t} / G A k$, $b=\left(E \operatorname{Im}_{r} / G A k+J_{t}\right) \pi^{2} / l^{2}+m_{r}, \quad c=E I \pi^{4} / l^{4}$ 。

根据式(24), 钢轨剪切刚度取 $2.11 \times 10^{8} \mathrm{~N}$, 转 动惯量 $J_{t}=0.2525 \mathrm{~kg} \cdot \mathrm{m}$, 依据本文轨道参数计算的 Euler 梁和 Timoshenko 梁钢轨的 pinned-pinned 共振 频率分别为 $1443 \mathrm{~Hz}$ 和 $1040 \mathrm{~Hz}$ ，前者与图 6 完全 一致, 后者略低于图 15 中轮轨力的 pinned- pinned 共振频率。

图 16 为车轮和钢轨振动加速度功率谱密度曲 线。两种速度时, 轮轨振动加速度的第 1 主频均为 P2 共振频率, 低于 P2 共振频率时, 车轮振动加速 度要高于钢轨, 且频率越低差异越大; 而高于 P2 共振频率时, 钢轨振动加速度高于车轮; 高于 pinned-pinned 共振频率时, 轮轨振动响应均显著下 降。从轮轨系统振动加速度的频响分布可知, 当频 率低于 $\mathrm{P} 2$ 共振频率时, 轮轨系统体现为车轮随动特 征，高于 P2 共振频率时，轨道振动占主导地位，当 频率继续增高时, 为惯性作用区, 轮轨系统振动不 显著。对比两种冲击速度的频响曲线, 较高的轮轨 冲击响应中, 轮轨系统均存在以 P2 共振频率为基频 的高次谐波振动, 尤其在轨枕振动活跃区, 车轮谐 波振动非常显著。

图 16 中还表明, 扣件支撑长度对轮轨系统加速 度主频分布影响不大，扣件支撑长度的增大会使 pinned-pinned 共振频率处轮轨振动加速度下降。

图 17 为车轮和钢轨位移响应功率谱密度曲线。 与振动加速度不同的是, 车轮和钢轨振动位移主频 均为 P2 共振频率, 考虑扣件离散支撑时轮轨位移在 


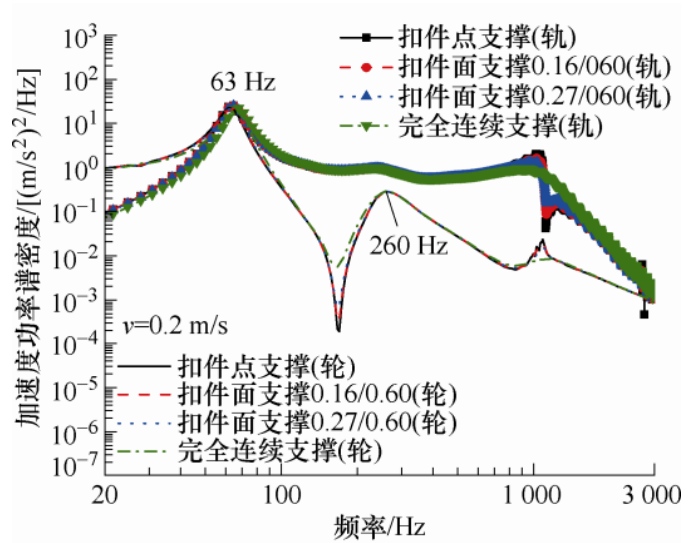

(a) 轮轨碰撞速度 $v=0.2 \mathrm{~m} / \mathrm{s}$

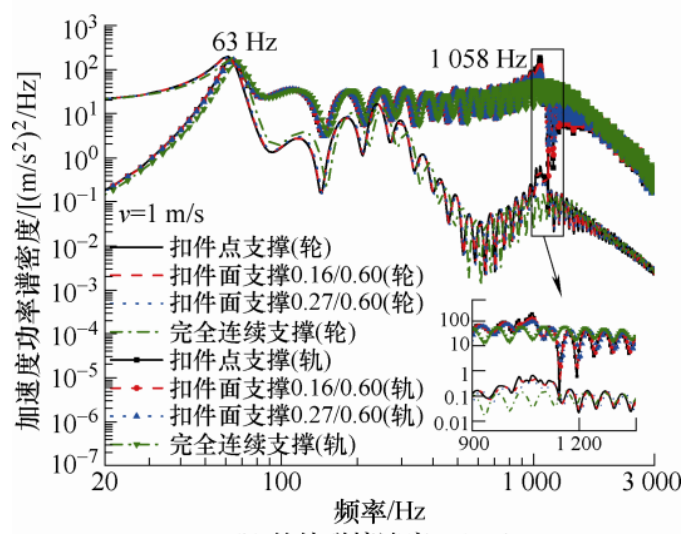

(b) 轮轨碰撞速度 $v=1 \mathrm{~m} / \mathrm{s}$

图 16 不同扣件支撑长度时轮轨加速度功率谱密度曲线

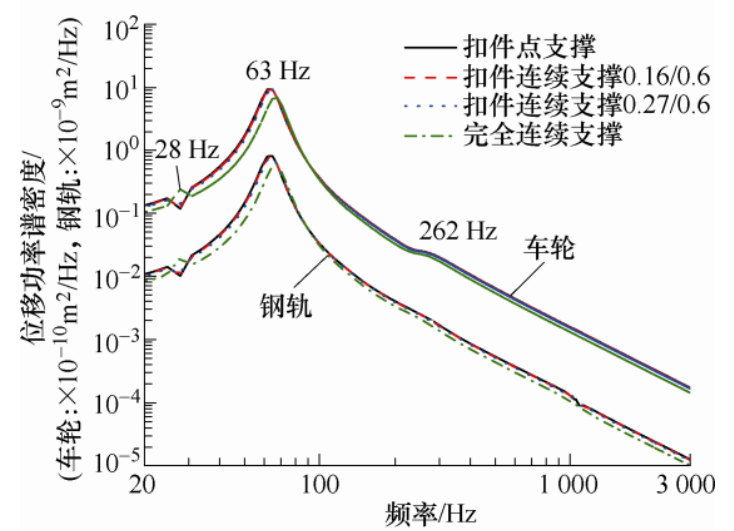

(a) 轮轨碰撞速度 $v=0.2 \mathrm{~m} / \mathrm{s}$

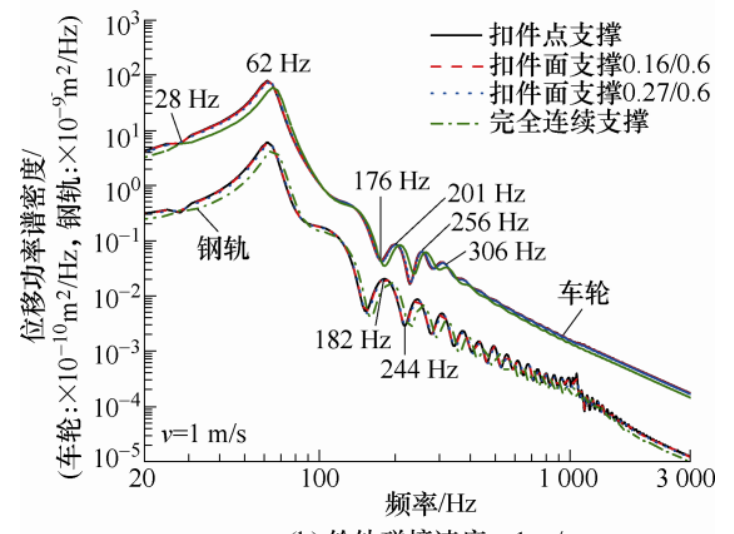

(b) 轮轨碰撞速度 $v=1 \mathrm{~m} / \mathrm{s}$

图 17 不同扣件支撑长度时轮轨位移功率谱密度曲线
$28 \mathrm{~Hz}$ 左右会出现轻微下降, 该频率为过轨跨频率。 冲击速度较低时, 车轮位移在 $260 \mathrm{~Hz}$ 附近略有上 升。当轮轨冲击速度较高时, 车轮在 $120 \sim 310 \mathrm{~Hz}$ 范围具有接近于 $\mathrm{P} 2$ 共振频率的谐波振动, 钢轨上以 P2 共振频率为基频等间隔频率分布的振动更为显 著。值得注意的是，不同于 P2 共振时轮轨具有相同 的主频, 在上述频率范围内, 车轮和钢轨振动主频 不一致, 如车轮在 $176 \mathrm{~Hz}$ 处于反共振峰, 而钢轨在 $182 \mathrm{~Hz}$ 时处于共振峰, 作为相互接触体的轮轨在相 近频率范围的响应特性差异对于其非均匀磨损具有 重要影响。图中还表明, 在 pinned-pinned 共振频率 处, 车轮振动位移没有变化, 较高冲击速度时, pinned-pinned 共振和反共振频率处的钢轨位移略有 波动。

\section{3 扣件支撑长度对轨枕振动频响的影响}

图 18 为轨道中心位置处轨枕振动加速度功率 谱密度曲线, 轮轨撞击速度为 $1 \mathrm{~m} / \mathrm{s}$ 。由图可知, 轨 枕振动加速度在 $68 \sim 200 \mathrm{~Hz}$ 频率范围内最为突出, 以 pinned-pinned 共振频率为中心, 在 $500 \sim 2000 \mathrm{~Hz}$ 范围内的轨枕振动加速度也较为显著, 在整个频率 范围内均能看到以 P2 共振频率为基频的高次谐波 频率影响。

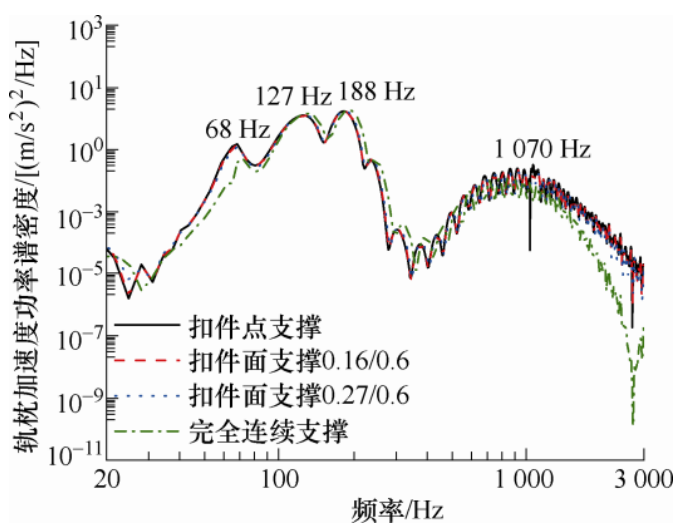

图 18 不同扣件支撑长度时轨枕振动响应曲线

\section{5 结论}

本文通过传递矩阵法分析了不同扣件支撑长度 的轮轨振动响应, 可以得出以下结论。

(1) 不考虑簧下质量耦合时, 轨道系统存在的 第 $1 、 2$ 共振频率分别对应于钢轨和扣件的同相共振 和反相共振; 高于 $1000 \mathrm{~Hz}$ 时, 当激振点位于跨中 时, 轨道系统存在明显的 pinned-pinned 共振频率, 激振点位于轨枕上方时, 钢轨出现反共振。其中, 第 1 、2 共振频率为轨道系统整体质量和刚度的振动 主频, 不受扣件支撑长度的影响。扣件支撑长度对 
pinned-pinned 共振和反共振影响显著, 随着扣件支撑 长度的增加, pinned-pinned 共振和反共振逐渐消失。

(2) 考虑簧下质量耦合后, 轮轨系统振动主要 以 P2 共振频率为主, 该频率显著低于轨道系统的第 1 自振频率, pinned-pinned 共振区的钢轨振动受扣 件支撑长度的影响显著, 而车轮和轨枕振动几乎不 受扣件支撑长度的影响。

(3) 轮轨撞击过程中的轮轨力以 $\mathrm{P} 2$ 共振频率为 基频波动, 撞击初期存在 $\mathrm{P} 1$ 高频波动。低于 $\mathrm{P} 2$ 共 振频率时, 车轮振动响应高于轨道, 高于 P2 共振频 率时, 轨道响应高于车轮, 当频率高于 $2000 \mathrm{~Hz}$ 时, 轮轨系统处于惯性主导区, 轮轨响应随频率提高而 迅速降低。

(4) 扣件支撑长度的增加可降低车轮和轨道在 pinned-pinned 共振区的加速度响应, 对车轮振动位 移影响不明显, 但可使平衡状态轮轨下沉量减小。 受滚动冲击载荷作用时, 轨枕的振动响应在 68 $200 \mathrm{~Hz}$ 范围内最为突出, 受扣件支撑长度影响不大。

(5) 本文研究表明, 扣件支撑长度的增加对降 低钢轨的 pinned-pinned 共振具有显著效果, 在条件 允许的情况下增加扣件的支撑长度可抑制或减缓 pinned-pinned 共振所致短波长波磨的萌生和发展。

（6）本文提出的考虑支撑长度的结构振动传递 矩阵分析方法可用于具有任意支撑长度的无限长周 期结构的振动特性分析。对于具有分段连续支撑的 有限长结构的振动特性分析, 仅需对边界条件进行 相应改变即可。

\section{参 考 文 献}

[1] KNOTHE K L, GRASSIE S L. Modelling of railway track and vehicle track interaction at high frequencies[J]. Vehicle System Dynamics， 1993， 22(3-4): 209-262.

[2] 翟婉明, 王其昌. 轮轨动力分析模型研究 $[\mathrm{J}]$. 铁道学 报，1994，16(1): 64-72.

ZHAI Wanming, WANG Qichang. A study on the analytical models for wheel/rail dynamics[J]. Journal of the China Railway Society, 1994, 16(1): 64-72.

[3] NIELSEN J C O, LUNDÉN R, JOHANSSON A, et al. Train-track interaction and mechanisms of irregular wear on wheel and rail surfaces[J]. Vehicle System Dynamics, 2003, 40(1-3): 3-54

[4] POPP K, KRUSE H, KAISER I. Vehicle-track dynamics in the mid-frequency range $[\mathrm{J}]$. Vehicle System Dynamics, 1999, 31(5-6): 423-464.

[5] CLARK R A, DEAN P A, ELKINS J A, et al. An investigation into the dynamic effects of railway vehicles running on corrugated rails[J]. Journal of Mechanical
Engineering Science, 1982, 24(2): 65-76.

[6] GRASSIE S L. Rail corrugation: characteristics, causes, and treatments[J]. Proceedings of the Institution of Mechanical Engineers, Part F: Journal of Rail and Rapid Transit, 2009, 223(6): 581-596.

[7] GRASSIE S L, GREGORY R W, HARRISON D, et al. The dynamic response of railway track to high frequency vertical excitation[J]. Journal of Mechanical Engineering Science, 1982, 24(2): 97-102.

[8] NORDBORG A. Wheel/rail noise generation due to nonlinear effects and parametric excitation[J]. Journal of the Acoustical Society of America, 2002, 111(4): 1772-1781.

[9] 赵悦, 肖新标, 韩健, 等. 高速有砟轨道钢轨动力吸振 器垂向吸振特性及其参数影响 $[\mathrm{J}]$. 机械工程学报, 2013, 49(16): 17-25.

ZHAO Yue, XIAO Xinbiao, HAN Jian, et al. Vertical characteristic and its parameter effect of rail vibration absorber used in high-speed ballasted track [J]. Journal of Mechanical Engineering, 2013, 49(16): 17-25.

[10] MAZILU T. Green's functions for analysis of dynamic response of wheel/rail to vertical excitation[J]. Journal of Sound and Vibration, 2007, 306(1-2): 31-58.

[11] 娄平, 曾庆元. 移动荷载作用下板式轨道的有限元分析 [J]. 交通运输工程学报，2004，4(1)：29-33.

LOU Ping, ZENG Qingyuan. Vertical characteristic and its parameter effect of rail vibration absorber used in high-speed ballasted track[J]. Journal of Traffic and Transportation Engineering, 2004, 4(1): 29-33.

[12] ZHANG J, GAO Q, TAN S J, et al. A precise integration method for solving coupled vehicle-track dynamics with nonlinear wheel-rail contact[J]. Journal of Sound and Vibration, 2012, 331(21): 4736-4773.

[13] ONO K, YAMADA M. Analysis of railway track vibration[J]. Journal of Sound and Vibration, 1989, 130(2): 269-297.

[14] ILIAS H. The influence of railpad stiffness on wheelset/track interaction and corrugation growth[J]. Journal of Sound and Vibration, 1999, 227(11): 935-948.

[15] EGANA J I, VINOLAS J, SECO M. Investigation of the influence of rail pad stiffness on rail corrugation on a transit system[J]. Wear, 2006, 261(2): 216-224.

[16] 谷爱军, 刘维宁, 张厚贵, 等. 地铁扣件刚度和阻尼对 钢轨异常波磨的影响 [J]. 都市快轨交通，2011，24(3): 17-21.

GU Aijun, LIU Weining, ZHANG Hougui, et al. Impact of rail fastenings' stiffness and damping on abnormal rail corrugation[J]. Urban Rapid Rail Transit, 2011，24(3): $17-21$. 
[17] 李霞, 李伟, 申芗芗, 等. 基于轨道振动理论的梯形轨 枕轨道钢轨波磨研究[J]. 机械工程学报, 2016, 52(22): 121-128.

LI Xia, LI Wei, SHEN Yingying, et al. Study on the rail corrugation of the ladder-type sleepers track based on the track vibration theory[J]. Journal of Mechanical Engineering, 2016, 52(22): 121-128.

[18] 王开云, 蔡成标, 朱胜阳. 铁路钢轨扣件系统垂向动力 模型及振动特性[J]. 工程力学, 2013，30(4): 146-149, 168.

WANG Kaiyun, CAI Chengbiao, ZHU Shengyang. Vertical dynamic model and vibration characteristic of rail fastening system[J]. Engineering Mechanics, 2013, 30(4): 146-149, 168.

[19] FERRARA R, LEONARDI G, JOURDAN F. A contact-area model for rail-pads connections in 2-D simulations : sensitivity analysis of train induced
vibrations[J]. Vehicle System Dynamics, 2013, 51(9): 1342-1362.

[20] BLANCO B, ALONSO A, KARI L, et al. Distributed support modelling for vertical track dynamic analysis[J]. Vehicle System Dynamics， 2018， 56(4): 529-552.

[21] ZHONG W X, WILLIAMS F W. Wave problems for repetitive structures and symplectic mathematics[J]. Proceedings of the Institution of Mechanical Engineers, Part C: Journal of Mechanical Engineering Science, 1992, 206(6): 371-379.

[22] 屈维德, 唐恒龄. 机械振动手册 [M]. 北京: 机械工业 出版社, 2000 .

QU Weide, TANG Hengling. Handbook of mechanical vibration[M]. Beijing: China Machine Press, 2000.

作者简介: 关庆华, 男, 1981 年出生, 博士。主要研究方向为车辆轨道 动力学与轮轨关系。

E-mail: guan_qh@163.com 